\begin{tabular}{c|c|c} 
Eiszeitalter u. Gegenwart & 40 & $107-110$ \\
& $3 \mathrm{Abb}$.
\end{tabular}

Hannover 1990

\title{
Ein mittelpaläolithischer Fundplatz in den Basiskiesen der Emscher-Niederterrasse bei Bottrop/Westfalen
}

\author{
RALF-W. Schmitz *)
}

Terraces, gravel, archaeological sites, fossil localities, artifacts, Paleolithic, Acheulian, Mammuthus trigontherii, teeths, Th/U, absolute age, Middle Pleistocene, Saalian

North Rhine-Westphalia, Rhine Westphalian Basin, TK 4507

\begin{abstract}
Kurzfassung: Beim Umbau des Rhein-Herne-Kanales zwischen 1956 und 1975 wurde bei Bottrop in den „Knochenkiesen" an der Basis der Emscher-Niederterrasse ein Siedlungsplatz des pleistozänen Menschen vom Bagger erfaßt. Bei dem Inventar des Platzes handelt es sich kulturell um ein Jungacheuléen, eine durch Faustkeile und blattförmige Schaber gekennzeichnete Formengruppe. Bis heute ist das Alter dieser Formengruppe (vorletzte oder frühe letzte Kaltzeit) im westlichen Mitteleuropa der Kernpunkt einer Kontroverse, entzündet am wichtigsten Jungacheuléen-Fundplatz Nordwestdeutschlands, Salzgitter-Lebenstedt (TODE 1982).
\end{abstract}

Das in Bottrop reichlich erhaltene Zahnmaterial von Mammuthus trogontherii eröffnete die Möglichkeit, mit naturwissenschaftlichen Datierungsmethoden dieser Frage nachzugehen.

Daran geknüpft ist der Versuch einer chronologischen Einstufung der Basiskiese der Emscher-Niederterrasse, deren Einordnung, meist in das frühe Weichselglazial, ebenfalls umstritten ist.

[A Middle Palaeolithic Site in the Base Gravels of the Emscher Lower Terrace near Bottrop/Westphalia]

Abstract: During the course of the Rhine-Herne Canal reconstruction project carried out between 1956 and 1975, a dredge unearthed a Pleistocene settlement situated in the base gravel "Knochenkiese" of the Emscher Lower Terrace near Bottrop. The objects found at the site have been culturally classified as Late Acheulian, a form group characterized by hand-axes and leaf-shaped scrapers. In the Western part of Central Europe, the age assignment of this form group (second or early last glacial period) has always been and still is a central point of controversy, inspired by the most important Late Acheulian site at Salzgitter-Lebenstedt (TODE 1982 ) in North-West Germany.

Thanks to the ample dental material from Mammuthus trogontherii preserved in Bottrop it has become possible to

*) Anschrift des Verfassers: R.-M. Schmitz M. A., Institut für Ur- und Frühgeschichte, Weyertal 125, 5000 Köln 41. investigate this question by way of scientific date establishing methods. Linked to this is an attempt at chronologically classifying the base gravels of the Emscher Lower Terrace whose age assignment - mostly to the early Weichselian glacial period - is also still disputed.

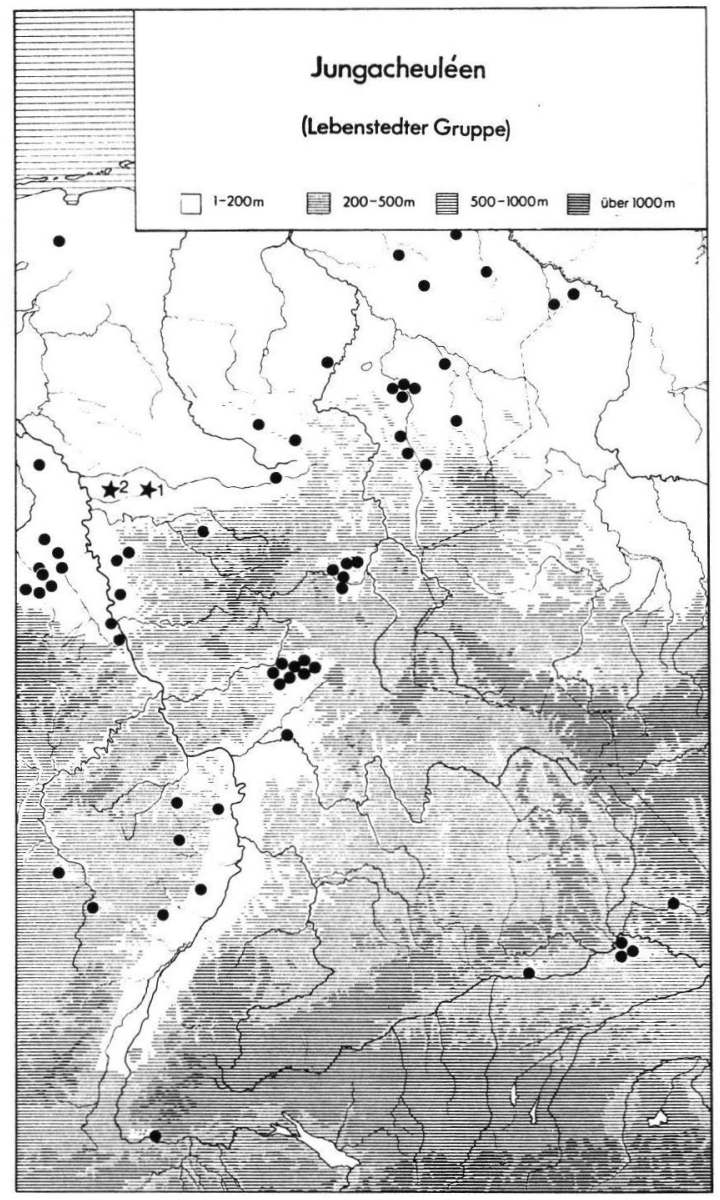

Abb. 1: Verbreitung des Jungacheuléen im westlichen Mitteleuropa (nach BosinSKI 1967). - 1 Herne. - 2 Bottrop. 


\section{Einleitung}

Seit Beginn des Jahrhunderts wurde beim Bau des Rhein-Herne-Kanals und bei späteren Wasserbaumaßnahmen im Emschertal häufig eine grobsandige bis kiesige Schicht angeschnitten, die nordisches Material und pleistozäne Großsäugerreste enthält.

Mehrfach wurden darin mittelpaläolithische Siedlungsplätze erfaßt, so in Herne (KAHRS 1912), Bottrop (HEINRICH 1980) und Essen-Dellwig (BOSINSKI 1983: 80f., 90). Die Fundschicht wurde als „Knochenkiese” bezeichnet - anfänglich deskriptiv nach ihrem Fundinhalt, später stratigraphisch, jedoch ohne Möglichkeit einer sicheren zeitlichen Einstufung.

Die Datierungsansätze schwanken zwischen Ende des Saaleglaziales (KAHRS 1912: 63), Übergang Saale/Eem (BÄRTLING 1912: 192), frühem Eem (MENZEL 1912: 200), spätem Eem bis frühem Weichselglazial (STEUSLOFF 1934) und frühem Weichselglazial (ANDREE 1928: 280).

Die „Knochenkiese” bilden nach BRUNNACKER (1982: 16, 26) die Älteste, nach JANSEN (1986: 102) einen Teil der Älteren Niederterrasse. Beide Autoren nehmen ein frühweichselzeitliches Alter der Ablagerungen an.

BOSINSKI (1963: 137-139; 1967: 41; 1982: 48) betont dagegen die Ähnlichkeit des paläolithischen Fundmateriales mit saalezeitlichen Funden aus Frankreich und der DDR, so z. B. im Liegenden der Drenthegrundmoräne in Markkleeberg bei Leipzig (BAUMANN \& MANIA 1983: 88). Einen chronologischen Anhaltspunkt in der ansonsten nicht exakt festlegbaren Fauna stellt der Schädelrest einer Saiga-Antilope (Saiga tatarica ssp) dar; KAHLKE (1975: 136) hält aufgrund der Morphologie eine Zuordnung des Bottroper Fundes zum Formenkreis der saalezeitlichen Saigas Europas für möglich.

\section{Der Fundplatz Bottrop}

Zwischen den Kanalkilometern 13,2 und 16,7 wurde der Rhein-Herne-Kanal in den Jahren 1956 bis 1975 (mit längeren Unterbrechungen) verbreitert und vertieft. Das anfallende Baggergut wurde mit Wasser vermischt und auf ein Spülfeld gepumpt, wo A. HEIN$\mathrm{RICH}$, der Leiter des Museums für Ur- und Ortsgeschichte Bottrop, und seine Helfer den Hauptteil des
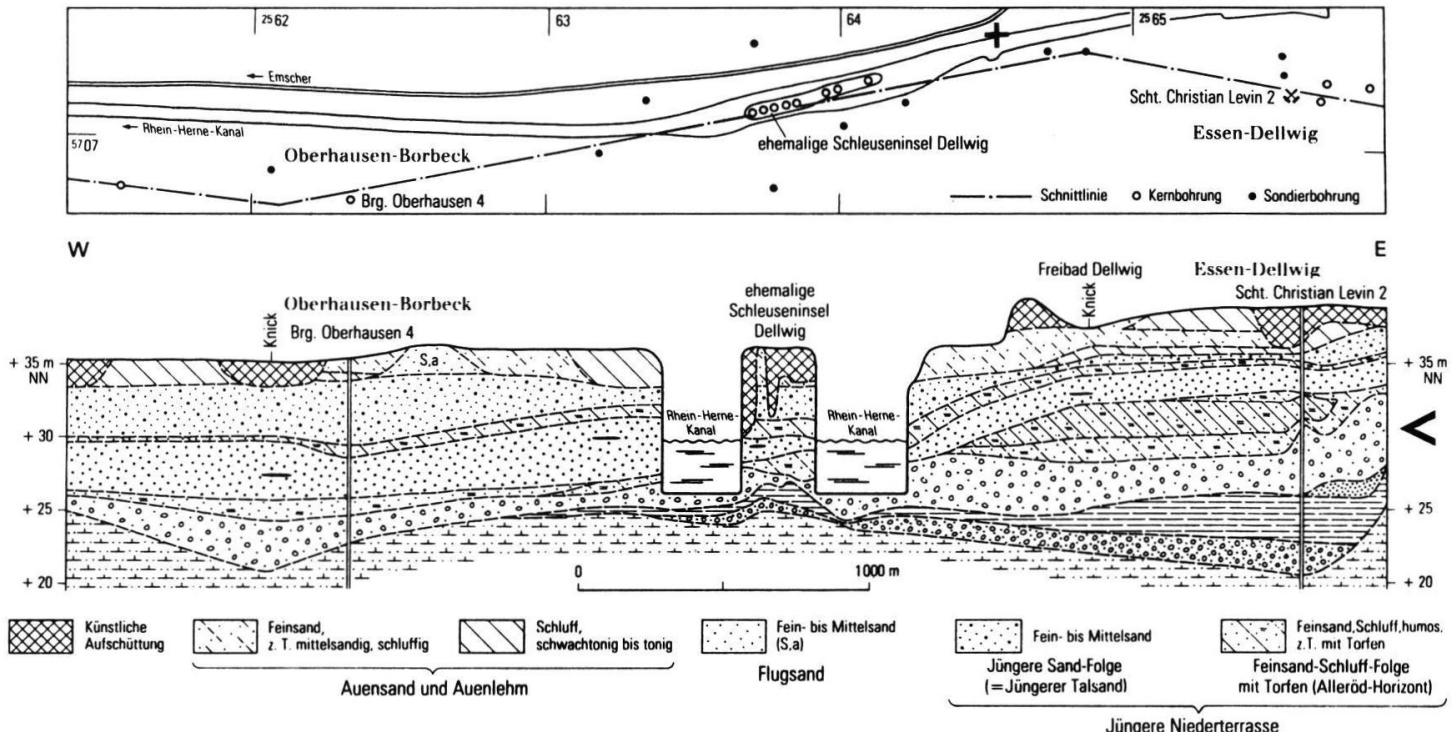

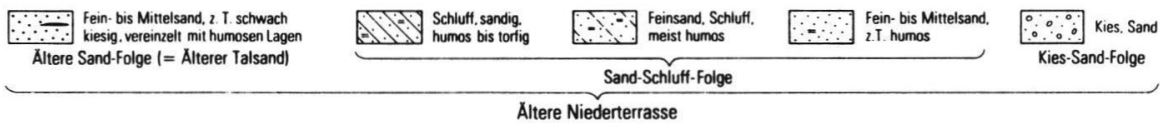

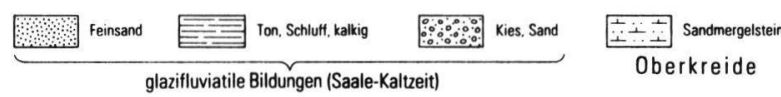

Abb. 2: Quartärablagerungen im Bereich Oberhausen-Borbeck/Essen-Dellwig/Bottrop. -

+ Fundplatz Bottrop, < Fundschicht. (Aus JANSEN \& DROZDZEWSKI 1986; Fundort/Fundschicht vom Verf. gekennzeichnet. 
Fundmateriales bergen konnten. Ein kleiner Teil der Funde wurde direkt auf dem Eimerkettenbagger sichergestellt. Steinartefakte und vom Menschen bearbeitetes faunistisches Material konnten nur dann beobachtet werden, wenn der Bagger bei Kanalkilometer 13,65 innerhalb eines 45 Meter langen $\mathrm{Ab}$ schnittes im nördlichen Kanaldrittel lag (HEINRICH 1987: 80, 131).

Der Vergleich der Baggertiefe mit Bohrprofilen ermöglichte die Zuweisung der Funde zu den „Knochenkiesen" (HEINRICH 1980: 113). Die Fundschicht entspricht der von JANSEN (1986: 104) beschriebenen Kies-Sand-Folge.

Es konnten 364 aus nordischem Feuerstein hergestellte Artefakte geborgen werden, die bis auf wenige Ausnahmen nicht verwittert oder abgerollt sind; daher
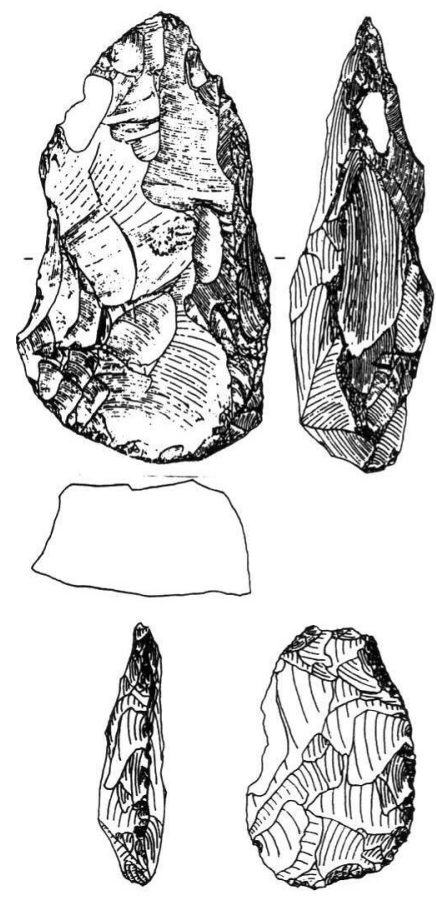

2
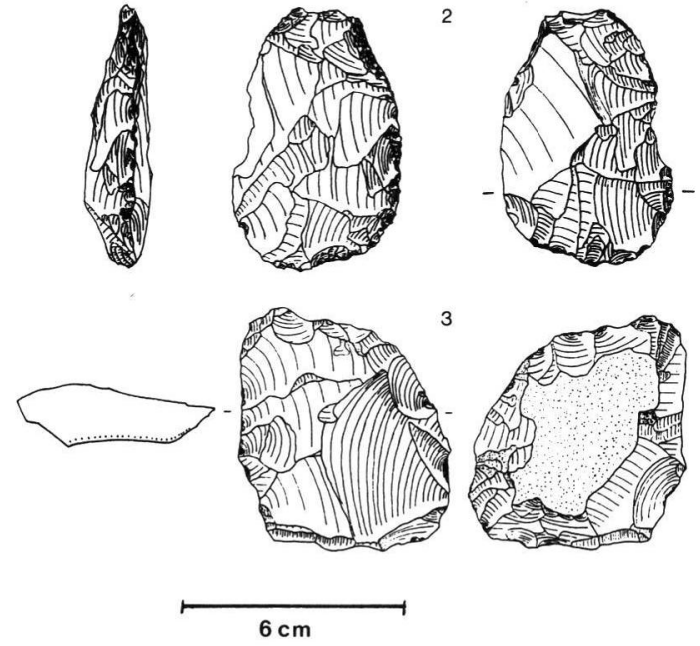

Abb. 3: 1 Faustkeil, 2 beidflächig retuschierter Schaber Typ Herne, 3 präparierter Kern vom Fundplatz Bottrop. - kann es als gesichert gelten, daß in Bottrop ein unverlagerter Siedlungsplatz entdeckt wurde.

Mit Faustkeilen, blattförmigen Schabern und präparierten Kernen (Abb. 3) sind die Kriterien für eine kulturelle Zuordnung zum Jungacheuléen gegeben.

Der sehr gute Erhaltungszustand der Steinartefakte erlaubte eine von G. LASS am Seminar für Ur- und Frühgeschichte der Universität Münster durchgeführte mikroskopische Gebrauchsspurenanalyse. Bei 200facher Vergrößerung zeigten die Silices ein breites Verwendungsspektrum: neben dem Zerlegen von Tieren konnte die Bearbeitung von $\mathrm{Holz}$, Knochen, Knochen/Geweih und Haut/Leder nachgewiesen werden.

Knochen, Geweih und Elfenbein ließen sich direkt durch bearbeitete Fundstücke als Werkstoffe belegen; so liegen u. a. Knochen- und Geweihstücke mit tief eingeschnittenen Kerben vor.

Das Tätigkeitsspektrum der Bottroper Freilandstation umfaßt somit alle Arbeiten, die man für den Bereich eines längerfristig besiedelten Platzes erwartet.

Nach den durch das Wasser- und Schiffahrtsamt Duisburg erstellten Bohrprofilen floß ein von $\mathrm{S}$ in die UrEmscher mündender Bach unmittelbar am Siedlungsplatz vorbei (mündl. Mitt. U. STOTTROP, Ruhrlandmuseum Essen); daraus ergibt sich eine für mittelpaläolithische Freilandstationen häufig beobachtete Lage im Winkel zwischen einem Fluß und einem Bachlauf.

\section{Datierung}

Den Knochenkiesen des Siedlungsplatzumfeldes entstammt eine große Anzahl pleistozäner Großsäugerreste (HEINRICH 1987).

Aus dem Mammuthus-Zahnmaterial wurden drei sehr gut erhaltene Molare von Mammuthus trogontherii ausgewählt und von R. HAUSMANN am Geologischen Institut der Universität Köln mit der Thorium/UranMethode datiert. Dabei ergaben sich Mittelwerte von 189.300, 144.100 und 126.900 Jahren B.P.

Da alle Daten vor das Eem fallen, liegt ein erster konkreter Beleg für das Alter des nordwestdeutschen Jungacheuléen und für die wa r the z e i t$1 \mathrm{i}$ c h e Einstufung der basalen Kiese der EmscherNiederterrasse vor.

Die Gleichsetzung der „Knochenkiese” des Emschertales mit ähnlichen Ablagerungen der Lippe erscheint nunmehr fraglich, da ${ }^{14} \mathrm{C}$-Datierungen von Knochen aus der Älteren Niederterrasse der Lippe konventionelle Alter von $36.600 \pm 1220$ und $41.910 \pm 960$ Jahren B.P. erbrachten (SKUPIN 1983: 48). 
Weitere Datierungen sind notwendig, auch im Hinblick auf die Frage, ob das Jungacheuléen eine reine Erscheinung des Saaleglaziales ist oder ob es ein Fortleben dieser Formengruppe in der frühen Weichselkaltzeit gibt.

\section{Schriftenverzeichnis}

ANDREE, J. (1927): Altsteinzeitliche Funde aus Westfalen III.: Das Aurignacien von Datteln (Westf.) und die Altersstellung der paläolithischen Funde von Lippe und Emscher. - Mannus, 19: 92-103, 4 Abb., 5 Tab.; Leipzig.

Baumann, W. \& Mania, D. (1983), mit Beitr. von Toeprer, V. und EISSMANN, L.: Die paläolithischen Neufunde von Markkleeberg bei Leipzig. - Veröff. Landesmuseums für Vorgeschichte Dresden, 16: 280 S., $192 \mathrm{Abb}$., 33 Taf., 9 Tab.; Dresden.

BÄRTLING, R. (1912): Über das geologische Alter der Funde von Menschenresten und Artefakten im niederrheinischwestfälischen Industriebezirk. - Z Ethnologie, 44: 187-195, 3 Abb.; Berlin.

BosINSKI, G. (1963): Eine mittelpaläolithische Formengruppe und das Problem ihrer geochronologischen Einordnung. - Eiszeitalter u. Gegenwart, 14: 124-140, 9 Abb., 1 Tab.; Öhringen/Württ.

- (1967): Die mittelpaläolithischen Funde im westlichen Mitteleuropa. - Fundamenta, A 4: XI + 206 S., XV + 197 Taf., 7 Kt.; Köln, Graz.

- [Hrsg.] (1982): Das Eiszeitalter im Ruhrland. - Ruhrlandmuseum Essen: 75 S., 46 Abb., 1 Tab., 2 Kt.; Köln.

- (1983): Die jägerische Geschichte des Rheinlandes. Jb. RGZM, 30: 81-112, 2 Tab.; Mainz.

BRUNNACKER, K. (1982): Geologie. - In: BOSINSKI, G. [Hrsg.]: Das Eiszeitalter im Ruhrland. - Ruhrlandmuseum Essen: 75 S., 46 Abb., 1 Tab., 2 Kt.; Köln.

HEINRICH, A. (1980): Eiszeitliche Funde aus dem RheinHerne-Kanal bei Bottrop. - Westfälische Geographische Studien, 36: 113-115; Münster.
HeINRICH, A. (1987): Geologie und Vorgeschichte Bottrops. - Geschichte Bottrops, I: 256 S., 191 Abb.; Bottrop (Historische Gesellschaft e. V.).

JANSEN, F. \& DRozDZewSki, G. (1986), mit Beitr. von Nötting, J., PaAs, W., Suchan, K. H. \& Vieth-RedeMANN, A.: Erläuterungen zu Blatt 4507 Mülheim a. d. R. - Geol. Kt. Nordrh.-Westf. 1:25000, Erl., 4507 Mülheim a.d. R.: 200 S., 18 Abb., 17 Tab., 4 Taf.; Krefeld (Geol. L.-Amt Nordrhein-Westfalen).

KAHLKE, H. D. (1975): Der Saiga-Fund von Bottrop/Westfalen. - Quartär, 26: 135-146, 1 Abb., 1 Taf.; Bonn.

KAHRS, E. (1912): Aus dem Museum der Stadt Essen: Prähistorische Funde im Industriegebiet. - In: Essens Entwicklung 1812-1912: 61-63, 1 Abb.; Essen (Fredebeul u. Koenen).

Menzel, H. (1912): Die Fauna der Fundschichten des quartären Menschen vom Rhein-Herne-Kanal und die Altersbestimmung der Artefakte. - Z. Ethnologie, 44: 195-200, 5 Abb.; Berlin.

SchmiTZ, R. W. (1988): Die mittelpaläolithischen Fundplätze Herne und Bottrop im Emschertal. - Archäologisches Korrespondenzblatt, 18: 311-321, 8 Abb., 3 Taf.; Mainz.

Skupin, K. (1983), mit Beitr. von Dahm-Arens, H., Michel, G., Rehagen, H. W. \& Vogeler, H.: Erläuterungen zu Blatt 4217 Delbrück. - Geol. Kt. Nordrh.-Westf. 1:25000, Erl., 4217 Delbrück: 120 S., 20 Abb., 6 Tab., 2 Taf.; Krefeld (Geol. L.-Amt Nordrhein-Westfalen).

Steusloff, U. (1934): Das Periglazial des Jungdiluviums im Emscher- und Lippetal. - Sitz.-Ber. naturhist. Ver. preuß. Rheinld. u. Westf., 1932/1933: 17-25, 1 Abb.; Bonn.

Tode, A. (1982): Der altsteinzeitliche Fundplatz SalzgitterLebenstedt. - Fundamenta, A 11/1, 71 S., 6 Abb., 138 Taf.; Köln, Graz.

Manuskript eingegangen am 25.9. 1989. 